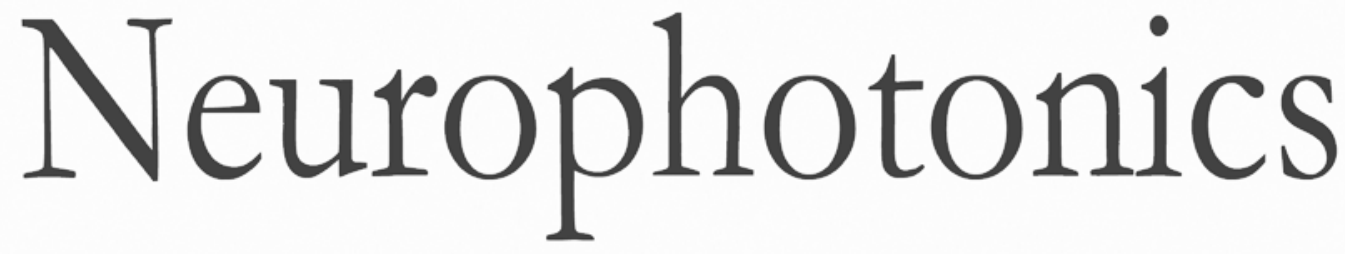

\title{
Quantitative confocal optical coherence elastography for evaluating biomechanics of optic nerve head using Lamb wave model
}

\author{
Zhaodong Du \\ Runze Li \\ Xuejun Qian \\ Gengxi Lu \\ Yan Li \\ Youmin $\mathrm{He}$ \\ Yueqiao Qu \\ Laiming Jiang \\ Zeyu Chen \\ Mark S. Humayun \\ Zhongping Chen \\ Qifa Zhou
}




\title{
Quantitative confocal optical coherence elastography for evaluating biomechanics of optic nerve head using Lamb wave model
}

\author{
Zhaodong $\mathrm{Du}^{\mathrm{a}, \mathrm{b}, \dagger}$ Runze $\mathrm{Li}^{\mathrm{b}, \mathrm{c}, \mathrm{c}, \dagger}$ Xuejun Qian, ${ }^{\mathrm{b}, \mathrm{c}, \dagger}$ Gengxi Lu, ${ }^{\mathrm{b}, \mathrm{c}}$ Yan $\mathrm{Li}^{\mathrm{d}, \mathrm{e}}$ Youmin He, ${ }^{\mathrm{d}, \mathrm{e}}$ Yueqiao Qu, ${ }^{\mathrm{d}, \mathrm{e}}$

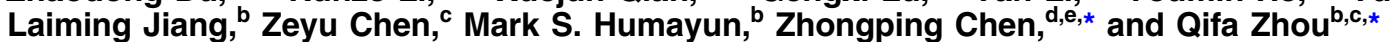 \\ ${ }^{a}$ The Affiliated Hospital of Qingdao University, Department of Ophthalmology, Qingdao, China \\ bUniversity of Southern California, Roski Eye Institute, Los Angeles, California, United States \\ 'University of Southern California, Department of Biomedical Engineering, Los Angeles, California, United States \\ dUniversity of California Irvine, Beckman Laser Institute, Irvine, California, United States \\ eUniversity of California Irvine, Department of Biomedical Engineering, Irvine, California, United States
}

\begin{abstract}
The mechanosensitivity of the optic nerve head $(\mathrm{ONH})$ plays a pivotal role in the pathogenesis of glaucoma. Characterizing elasticity of the $\mathrm{ONH}$ over changing physiological pressure may provide a better understanding of how changes in intraocular pressure (IOP) lead to changes in the mechanical environment of the ONH. Optical coherence elastography (OCE) is an emerging technique that can detect tissue biomechanics noninvasively with both high temporal and spatial resolution compared with conventional ultrasonic elastography. We describe a confocal OCE system in measuring ONH elasticity in vitro, utilizing a pressure inflation setup in which IOP is controlled precisely. We further utilize the Lamb wave model to fit the phase dispersion curve during data postprocessing. We present a reconstruction of Young's modulus of the ONH by combining our OCE system with a Lamb wave model for the first time. This approach enables the quantification of Young's modulus of the $\mathrm{ONH}$, which can be fit using a piecewise polynomial to the corresponding IOP. $\odot$ The Authors. Published by SPIE under a Creative Commons Attribution 4.0 Unported License. Distribution or reproduction of this work in whole or in part requires full attribution of the original publication, including its DOI. [DOI: 10.1117/1.NPh.6.4.041112]
\end{abstract}

Keywords: optical coherence elastography; acoustic radiation force; optic nerve head; Lamb wave model.

Paper 19061SSR received Jun. 18, 2019; accepted for publication Oct. 30, 2019; published online Nov. 15, 2019.

\section{Introduction}

As an optic neuropathy, glaucoma is one of the leading causes of blindness worldwide. ${ }^{1,2}$ Glaucomatous damage to the visual system likely includes important pathophysiologies within the photoreceptors, retinal ganglion cell (RGC), lateral geniculate body, and visual cortex. Evidence has shown that damage to the RGC axons within the lamina cribrosa (LC) of the optic nerve head $(\mathrm{ONH})$ is a central pathophysiology. ${ }^{3}$

The ONH is a region of special biomechanical interest because it is a discontinuity in the cornea-scleral shell, and this type of discontinuity typically gives rise to stress or strain concentrations in mechanical systems. ${ }^{4,5}$ The mechanical properties of the $\mathrm{ONH}$ are dependent on the interaction of its individual components. At low stress, it is likely that the elastin component allows for an initially large volume change formed by the distending $\mathrm{ONH}$ and, as pressure increases and the extended collagen fibers limit further deformation, the $\mathrm{ONH}$ becomes progressively stiffer. ${ }^{6}$ Elevated intraocular pressure (IOP) is the most important risk factor for glaucoma progression, ${ }^{7}$ which distorts the tissues of the $\mathrm{ONH}$, and $\mathrm{LC}$ within, triggering events, such as compromised axoplasmic flow, vascular perfusion, and astrocyte activation that eventually lead to optic nerve axon degeneration and RGC death. ${ }^{7,8}$ An improved understanding of the $\mathrm{ONH}$ biomechanical environment and of the dependence

\footnotetext{
*Address all correspondence to: Zhongping Chen, E-mail: z2chen@uci.edu; Qifa Zhou, E-mail: qifazhou@usc.edu

tThese authors contributed equally to this work and should therefore be
} regarded as equivalent authors. of this environment on the biomechanical properties of the $\mathrm{ONH}$ tissues is necessary to understand better how biomechanical effects may play a role in glaucomatous optic neuropathy. ${ }^{9}$

There are several experimental techniques currently being used to investigate changes in the mechanical compliance of the ONH and posterior scleral surface. ${ }^{10,11}$ Most of these identify the pressure-induced deformation at the level of the LC and use this information to determine the biomechanical properties of the tissue. ${ }^{10}$ However, it is known that the $\mathrm{ONH}$ and anterior laminar surfaces are not only displaced posteriorly but also anteriorly (with reference to Bruch's membrane opening) in a significant portion of glaucoma patients. ${ }^{12}$ Characterizing elasticity of the ONH over physiological pressures may provide a better understanding of how changes in IOP lead to changes in the mechanical environment of the ONH. ${ }^{13-15}$

Optical coherence elastography (OCE) is an emerging technique, which can detect tissue biomechanics noninvasively with both high temporal and spatial resolution compared with conventional ultrasonic elastography. ${ }^{16}$ The axial displacement range is typically in the micrometer to nanometer level. Recent work on advanced ultrasonic elastography demonstrated excellent spatial resolution with micrometer axial displacement detection. ${ }^{17,18}$ OCE shows great potential to assess the biomechanics of the posterior segment of the eye. In ophthalmology, however, this technique is mainly focused on the anterior segment of the eye, such as the cornea and the lens. ${ }^{19-23}$ Some OCE systems utilize oblique incidence air pulses ${ }^{20,24}$ or an obliquely oriented transducer ${ }^{25,26}$ to induce tissue vibration, which may generate complex waves instead of pure waves and can lead to errors in the estimation of biomechanical parameters. ${ }^{27} \mathrm{We}$ 
recently reported a confocal acoustic radiation force (ARF) OCE method that can map out the elasticity of retinal layers with high resolution. ${ }^{28}$ This confocal setup avoids generating complex waves, moreover, it allows for easy access to the retinal layers. However, due to the complexity of the retina, only group velocity was used to estimate the biomechanics of normal retina and age-related macular degeneration-induced retina.

One of the challenges in elastography is to reconstruct the biomechanics accurately. ${ }^{29}$ Group velocity has long been used to reconstruct the biomechanics, however, this method assumes that the elastic wave propagates in a homogeneous, isotropic, bulk, and purely elastic medium. ${ }^{30}$ In most tissues, elasticity and viscosity exist at the same time. Moreover, some tissues such as the ONH exhibit a thin plate morphology, which leads to a failure of biomechanics estimation using group velocity.

In our previous work, we demonstrated the feasibility of a Lamb wave model to quantify the viscoelasticity of imaging phantoms, porcine corneas as well as rabbit arteries. ${ }^{31}$ In this study, we investigate the ability of OCE for measurement of the ONH elasticity in pig eyes, utilizing a pressure inflation setup in which IOP is controlled precisely. We further utilize the Lamb wave model to fit the phase dispersion curve during our data postprocessing procedure. To our knowledge, this is the first time that a Lamb wave model has been used in evaluating the biomechanics of the ONH. Our aim was to quantify the mechanical behavior of the $\mathrm{ONH}$ in response to elevated IOP.

\section{Methods and Results}

\subsection{Preparation of Specimens}

Twenty porcine eyes were obtained from a U.S. Department of Agriculture-approved slaughterhouse. The mixed-breed pigs were all healthy and between 5 and 7 months old before they were killed. The eyes were immersed in saline and kept cold during shipping, and all eyes were tested within $48 \mathrm{~h}$ postmortem. The extraocular tissues were removed, and the optic nerve was cut to be flush with the outer surface of the peripapillary sclera by using a surgical blade. At the $\mathrm{ONH}$, the axons converge into bundles to pass through the LC, which spans the scleral canal and is continuous with the adjacent peripapillary sclera. Viewed from the back of eyeball, the ONH was surrounded by peripapillary sclera at the same level after the optic nerve was removed. Then, the entire eyeball was physically stabilized in a custom holder with the ONH facing upward. Two ports were created in the pars plana using $23 \mathrm{G}$ trocars (BL5523WVX, Bausch \& Lomb, Inc., Rochester, New York). One connected to a pressure column containing phosphatebuffered saline (PBS) to control IOP, and the other connected with an IOP sensor (Model SPR-524, Millar, Inc., Texas). Realtime readout of the IOP was performed using a custom-build LabView program, which provided feedback on the instantaneous IOP. The height of the infusion line was adjusted accordingly. The sample was then put in a PBS bath, which served as a medium for ultrasound propagation and a preservative for ocular tissue. The IOP was increased by manually raising the height of the PBS column from 10 to $30 \mathrm{mmHg}$. We used a pressure increment of $5 \mathrm{mmHg}$ for each step, and the IOP was held constant at each level for $1 \mathrm{~min}$ before the data were acquired.

\subsection{System Setup}

To assess the biomechanical properties of the ONH, a customized OCE system combining an ultrasonic transducer and a spectral-domain optical coherence tomography (SD-OCT) system was developed, as shown schematically in Fig. 1(a). In the SD-OCT system, a light beam was emitted from a superluminescent diode (SLD, M-D-890-HP1, Superlum Diodes Ltd, Carrigtwohill, Ireland) with a central wavelength of $890 \mathrm{~nm}$ and a bandwidth of $144 \mathrm{~nm}$ to detect tissue structural information as well as response to ultrasonic ARF. The light beam was filtered through an optical isolator (IO-F-SLD150-895, Thorlabs, Inc., Newton, New Jersey) to prohibit backprojection of light into the SLD and then split by an 80/20 optical coupler for efficient and safe data collection. In the reference arm, the light beam was collimated, attenuated, and reflected. In the sample arm, light was collimated, scanned through a galvanometer (GVS002, Thorlabs Inc.), and then focused via a 54-mm scan lens (LSM04-BB, Thorlabs Inc.) through the hollow part of the ring transducer to the tissue. The backscattering signal from the sample arm was interfered with the reflected signal from the reference arm and transmitted to the detecting arm. This

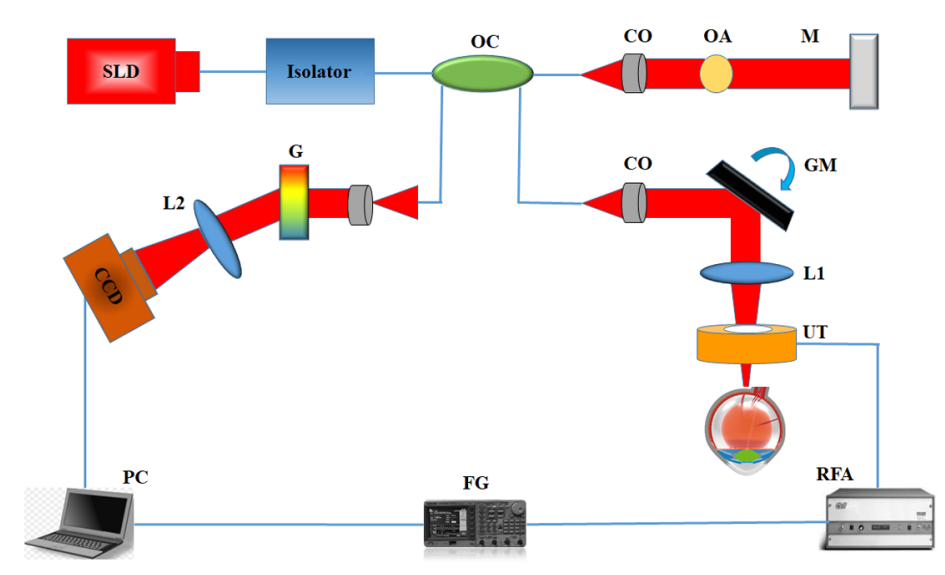

(a)

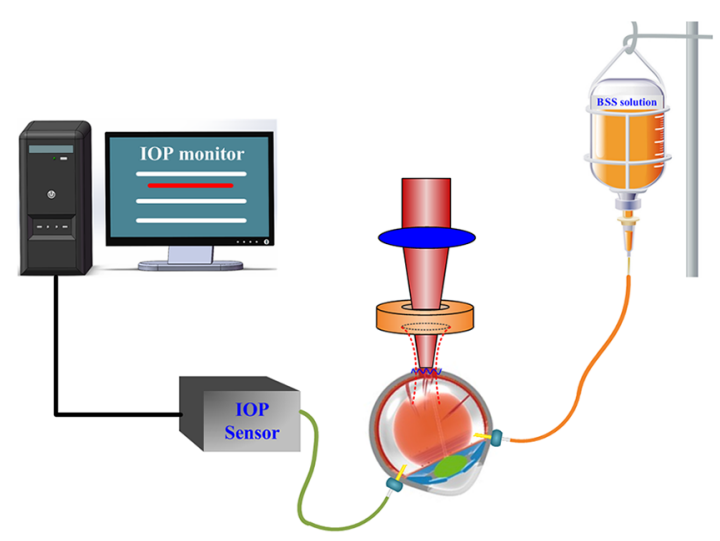

(b)

Fig. 1 (a) Optoacoustic elastography setup with unscalded porcine eye. SLD, superluminescent diode; $\mathrm{OC}$, optical coupler; $\mathrm{CO}$, collimator; $\mathrm{OA}$, optical attenuator; $\mathrm{M}$, mirror; $\mathrm{GM}$, galvanometer mirrors; L1/L2: lens; UT, ultrasonic transducer; RFA, radiofrequency amplifier; FG, function generator; and $G$, grating. (b) Schematic of the IOP control and measurement system. 
interference signal was split by wavelength via a diffraction grating and finally detected by a line scan CCD camera (spL4096$140 \mathrm{~km}$, Basler AG, An der Strusbek 60-62, Germany) at a $50-\mathrm{KHz}$ A-line rate. The signal was further processed to OCT intensity images and phase-resolved displacement maps. The axial resolution of this SD-OCT is around $3 \mu \mathrm{m}$ in air.

A custom-built $5 \mathrm{MHz}$ modified PZT material ring transducer was used to generate tissue motion. The aperture size is $23 \mathrm{~mm}$ with a $f$-number of 1 . The peripapillary sclera was selected as the ultrasonic excitation area, where uniform distribution of acoustic output initiated the propagation of elastic waves in the tissue. The detected displacement of the $\mathrm{ONH}$ was attributed to the response of this generated ARF. The excitation pulse was limited to $2 \mathrm{~ms}$ to allow the tissue to respond to the ARF naturally and recover to its original position before the next ultrasonic excitation. Confocal alignment of the ultrasound transducer and the SD-OCT system was achieved by using hydrophone (HGL-0085, ONDA Co, Sunnyvale, California) guidance before the experiment.

To acquire the OCT intensity images and phase-resolved displacement maps, a global clock was used to synchronize all subunits of the overall imaging system, including the ultrasound transducer, the CCD imager, and the SD-OCT, as shown in Fig. 2. ARF was generated with the galvanometer set to the P0 location and then, the elastic wave was free to propagate along with the imaged object. M-mode raw data were acquired at each of the galvanometer locations (e.g., P0, P1, P2, etc.) for 400 A-lines resulting in a total location interval of $8.8 \mathrm{~ms}$ ( $0.02 \mathrm{~ms}$ delay in every A-line for data storage). The step size for consecutive lateral positions was $4 \mu \mathrm{m}$ with 1000 lateral points in total. Then, the raw data were saved to disk for offline processing.

\subsection{Lamb Wave Model}

Young's modulus can be estimated simply in free space using the following set of equations: $:^{17}$

$\mu=\rho c_{g}^{2}, \quad E \approx 3 \mu$.
The shear modulus $(\mu)$ can be obtained using the density $(\rho)$ and constant group velocity $\left(c_{g}\right)$. Young's modulus $(E)$ is approximately three times the shear modulus. One of the assumptions above also includes a pure elastic medium for guided wave propagation. However, the $\mathrm{ONH}$ exhibits a thin-layer structure and is small compared with the shear wavelength. Given that Lamb waves typically travel in thin plates with a fluid boundary interface and ARF is applied orthogonally, a Lamb wave is identified as the guided wave propagated in the ONH. The speed of Lamb waves is dispersive and they can only propagate in the low-frequency range due to the viscosity in soft tissue. Since ARF was generated vertically from top to bottom through the thickness of the fluid column, with particles oscillating in the same direction, antisymmetric Lamb waves are mainly induced. Moreover, the zeroth order mode can travel at any frequency and is most commonly detected in the low-frequency range as compared with nonzeroth order mode Lamb waves. For a thin plate submerged in an incompressible fluid, the dispersion equation based on the zeroth order antisymmetric Lamb wave mode is as follows: $:^{31}$

$$
\begin{gathered}
4 k_{L}^{3} \beta_{L} \cosh \left(k_{L} h\right) \sinh \left(\beta_{L} h\right) \\
-\left(k_{s}^{2}-2 k_{L}^{2}\right)^{2} \sinh \left(k_{L} h\right) \cosh \left(\beta_{L} h\right) \\
=k_{s}^{4} \cosh \left(k_{L} h\right) \cosh \left(\beta_{L} h\right)
\end{gathered}
$$

where $\beta_{L}=\sqrt{k_{L}^{2}-k_{s}^{2}}, k_{L}=\omega / c_{L}$ is the Lamb wave number, $\omega$ is the angular frequency, $c_{L}$ is the frequency-dependent phase velocity, $k_{s}=\omega \sqrt{\rho / U}$ is the shear wavenumber, $U=$ $\mu+i \omega \eta$ is the shear modulus, $\mu$ and $\eta$ are shear elasticity and shear viscosity, respectively, and $h$ is the half thickness of the $\mathrm{ONH}$.

\subsection{Postprocessing}

The OCT intensity and Doppler phase raw data were first obtained, the spatial-temporal map along with lateral positions and time was then acquired by averaging the axial displacements

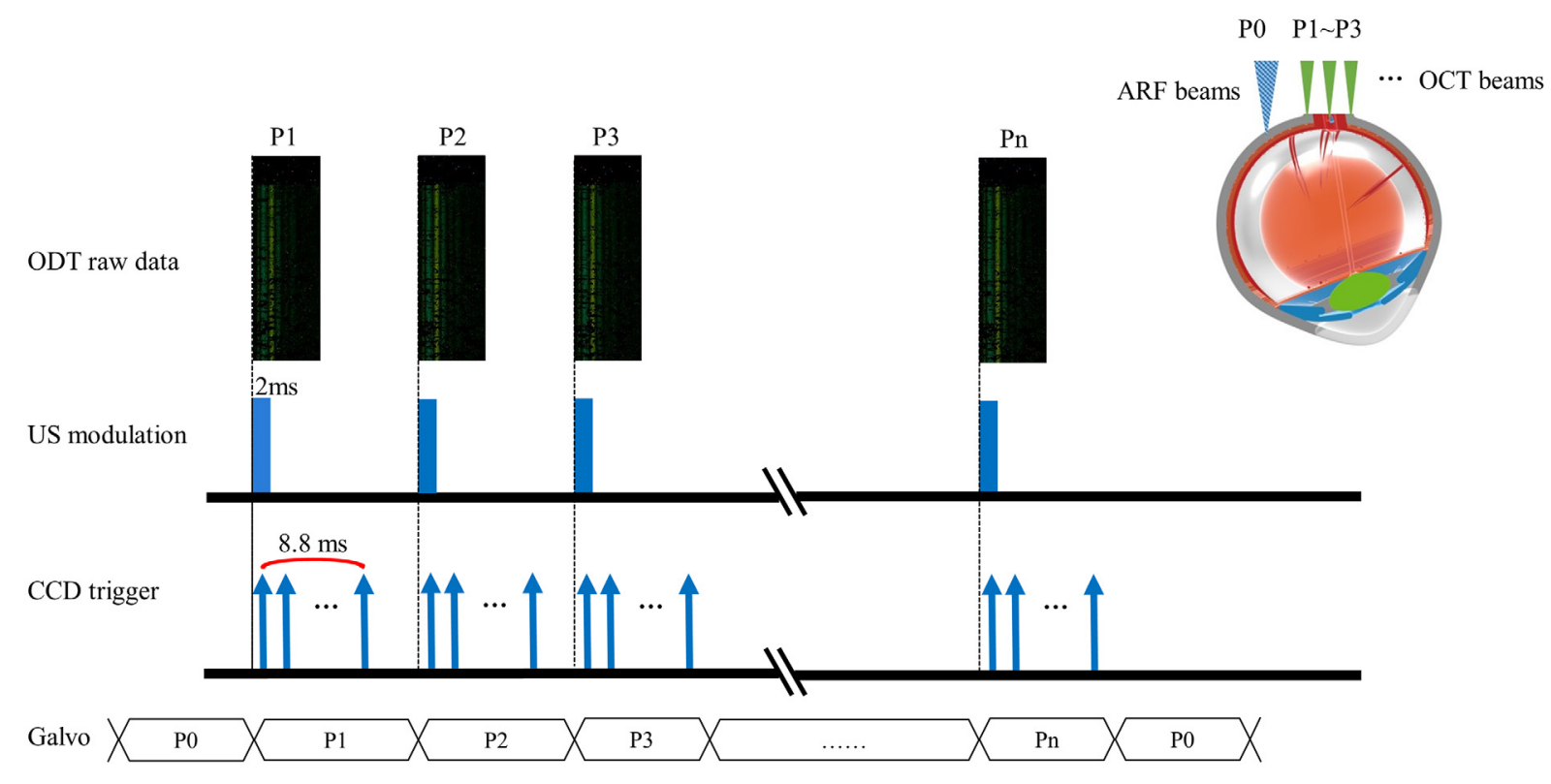

Fig. 2 Schematic representation for synchronization of the complete imaging system for synchronized confocal OCE. M-mode displacement map data were acquired in each location (e.g., P0, P1, P2, etc.). 
through the entire thickness of the ONH. The ultrasonic excitation regions in the field-of-view were all within the full width at half maximum axial intensity of acoustic field. The group velocity was obtained by applying linear regression to the wave crest at different lateral positions. The spatial-temporal map was then transformed to frequency/wavenumber domain $(k-$ space map) by using the two-dimensional (2-D) fast Fourier transform (FFT). The $k$-space map below $5 \mathrm{~Hz}$ was masked to avoid low-frequency noise. The phase velocity was acquired by dividing the frequency by the corresponding maximum wavenumber as follows:

$c_{L}=2 \pi f / k_{L}$.

The phase velocity was sampled up to $1200 \mathrm{~Hz}$ in $125-\mathrm{Hz}$ increment. Then, the shear modulus and viscosity-related fitted phase velocity were selected to minimize the error in every sampled phase velocity $R_{2}$ using the following cost function: ${ }^{31}$

$R^{2}=\sum\left[c_{f}(\mu, \eta)-c_{L}\left(f_{1}, f_{2}, \ldots, f_{n}\right)\right]^{2}$,

where $c_{f}$ is the curve fitted phase velocity, $f_{1}, \ldots, f_{n}$ are sampling frequencies with $125-\mathrm{Hz}$ interval. The error rate was also calculated to evaluate the precision of the fitting procedure by using experimental data and fitted data at every sampled frequency: ${ }^{31}$

$\mathrm{ER}=$ Average $\left(\frac{\sqrt{\left(c_{f}(f)-c_{L}(f)\right)^{2}}}{c_{L}(f)}\right)$

\section{Results}

\subsection{Calibration of the Imaging System}

The acoustic field of the 5-MHz ultrasonic transducer was measured using a needle hydrophone aligned to the focal point of the transducer. The measured $-6 \mathrm{~dB}$ lateral beamwidth was $384 \mu \mathrm{m}$ and the $-6 \mathrm{~dB}$ axial focal distance was $3.84 \mathrm{~mm}$, which can cover the OCT imaging depth in the ONH $(\sim 0.4 \mathrm{~mm})$ and enable an even distribution of acoustic intensity during data acquisition. In a previous study, the reconstructed Young's modulus of an agar imaging phantom using group velocity was found to be comparable with mechanical testing. ${ }^{32}$ We also previously demonstrated the feasibility of reconstructing Young's modulus of rabbit cornea and the posterior segment of the eye in vivo. ${ }^{28,33-35}$ With respect to the Lamb wave model, we previously showed the accuracy of this model in calculating Young's modulus in a thin and viscoelastic gelatin phantom as well as for corneal and arterial tissues. ${ }^{31}$

\subsection{ONH Response to Elevated IOP}

The biomechanical response of the ONH to elevated IOP was investigated with our Lamb wave model. After each data acquisition, OCT intensity and phase-resolved Doppler OCT data were acquired. Figure 3(a) shows the OCT intensity image of the posterior segment of the eye. By reslicing the ODT raw data [Figs. 3(b)-3(d)], the elastic wave is shown as propagating along this area. Figures 4(a)-4(d) show results on each postprocessing procedure. By averaging over the entire thickness direction of axial displacements along with lateral positions and time, the spatial-temporal map was generated. The wave crest in different lateral positions exhibits a linear trend, and the group velocity can be estimated using a linear regression algorithm. The 2-D FFT of the spatial-temporal map was then used to generate the $k$-space map as a function of frequency and wavenumber. The phase velocity curve was then obtained using Eq. (3), with the frequency at each point divided by the maximum intensity at that frequency. Due to the nature of viscosity in the $\mathrm{ONH}$, the phase velocity is dispersive, as shown in Fig. 4(d). The frequency range was up to $1200 \mathrm{~Hz}$. Finally, the phase velocity curve was sampled and fitted to the Lamb wave model using Eq. (2) with minimized error [Eq. (4)].

Figures 5(a)-5(c) show the sampled phase velocity and curve fitting results, as described above for a range of IOP levels, where IOP $=10,20$, and $30 \mathrm{mmHg}$, respectively. The experimental data match the curve-fit result with good agreement, and the average error rates (ERs) are from $5.4 \%$ to $11.09 \%$ when the IOP is below $30 \mathrm{mmHg}$. Young's modulus can be obtained by this curve-fitting procedure and is shown in Fig. 6: $181.3 \pm$ $21.0 \mathrm{kPa}$ at IOP of $10 \mathrm{mmHg}, 210.0 \pm 22.5 \mathrm{kPa}$ at IOP of $15 \mathrm{mmHg}, 231 \pm 31.6 \mathrm{kPa}$ at IOP of $20 \mathrm{mmHg}, 324.8 \pm$ $41.3 \mathrm{kPa}$ at IOP of $25 \mathrm{mmHg}$, and $450.5 \pm 52.5 \mathrm{kPa}$ at IOP of $30 \mathrm{mmHg}$, respectively. Young's modulus of the $\mathrm{ONH}$ can be curve-fit to the corresponding IOP using a piecewise polynomial. The goodness-of-fit $R^{2}$ was $99.7 \%$, which suggests that it should be possible to evaluate Young's modulus with higher IOP in future work.

\section{Discussion}

In this paper, we have presented reconstruction of the Young's modulus of the ONH by combining our OCE system with a Lamb wave model for the first time. The high spatial and temporal resolution of our OCE system enables the acquisition of a highly sensitive spatial-temporal map, which enables high accuracy for subsequent postprocessing. The confocal configuration of the transducer with OCT allows the ARF to be applied to the imaging object orthogonally, which avoids generation of complex elastic waves, such as Love waves, which increases the accuracy of biomechanical estimation. The confocal

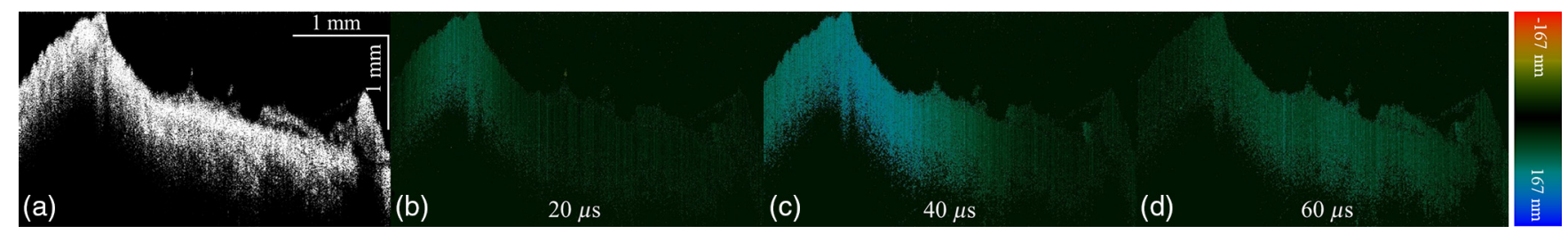

Fig. 3 (a) The OCT intensity image of the posterior segment of the eye. (b)-(d) A time series of ODT raw data shows the elastic wave propagating across the $\mathrm{ONH}$. Axial displacement is related to color, as shown in the color bar at the right of the figure. 

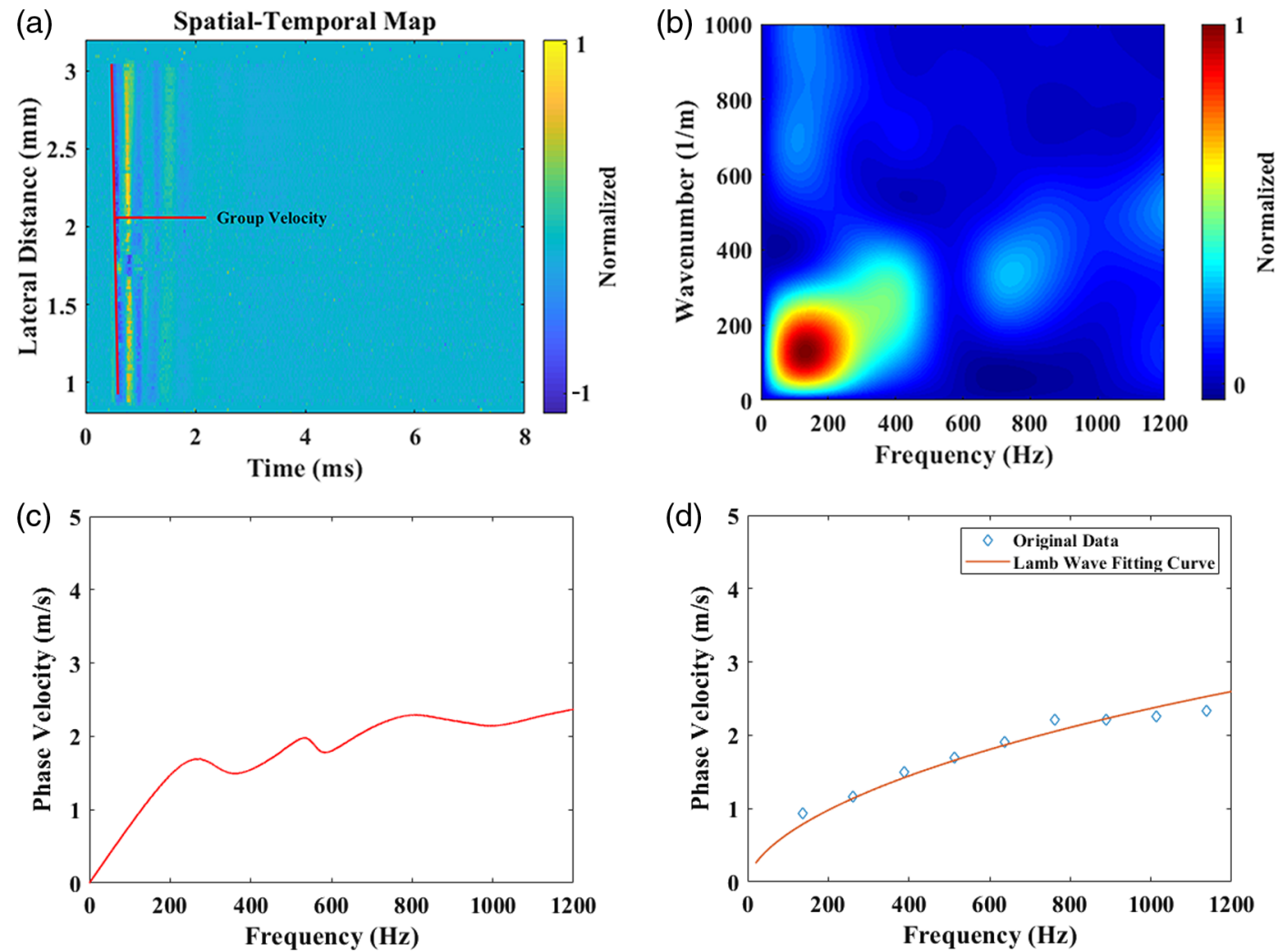

Fig. 4 Data postprocessing flow during each biomechanics assessment with curves generated by averaging axial displacement through the entire thickness with lateral positions: (a) spatial-temporal map, (b) k-space by 2-D FFT, (c) frequency normalized by corresponding maximum wavenumber, (c) phase velocity, and (d) curve fit in the frequency of 0 to $1200 \mathrm{~Hz}$ in $125-\mathrm{Hz}$ increments.

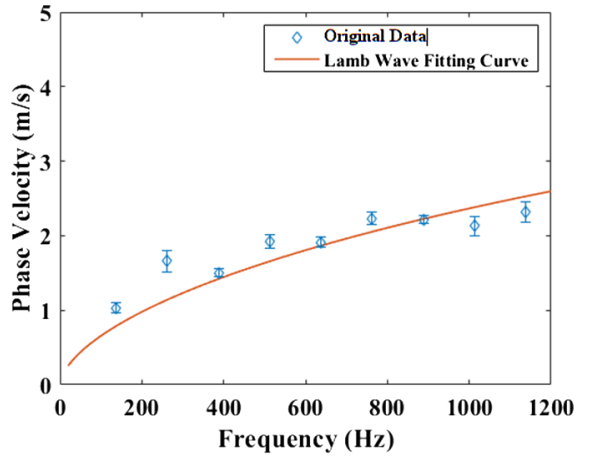

(a)

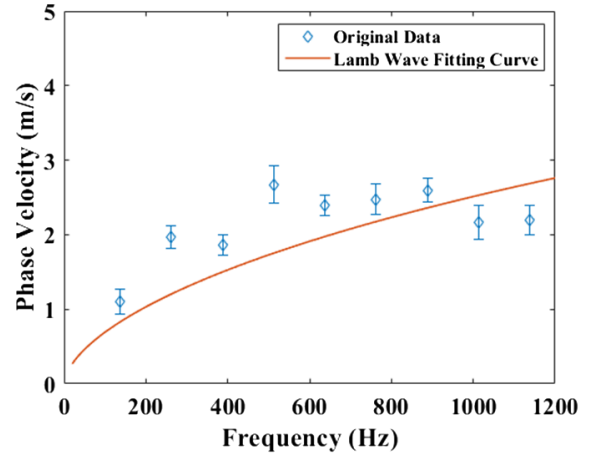

(b)

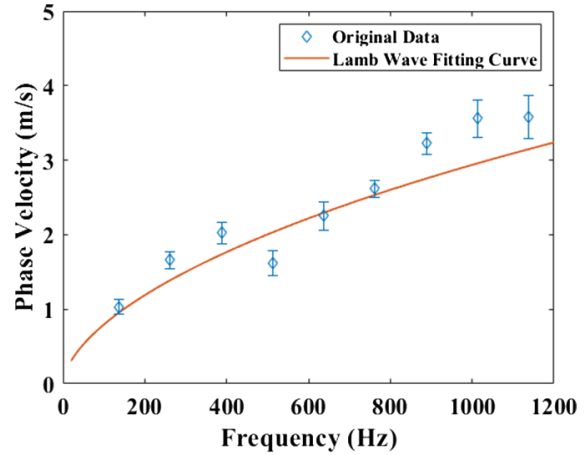

(c)

Fig. 5 Experimental phase velocity (diamond symbols) and corresponding Lamb wave model curve fit with different IOP levels: (a) $10 \mathrm{mmHg}$, (b) $20 \mathrm{mmHg}$, and (c) $30 \mathrm{mmHg}$. Error bars represent the standard deviation between experimental data and fitted curve at each sampled frequency.

configuration also allows the OCT beam to penetrate through the center hole in the ultrasonic transducer without incurring significant attenuation, which should enable us to assess the biomechanics of the $\mathrm{ONH}$ in vivo for future studies. We further introduced a Lamb wave model in this study. By fitting the experimental phase velocity to the Lamb wave model with low $\mathrm{ER}$, the biomechanics of the $\mathrm{ONH}$ can be reconstructed with elevated IOP.

Previous work by other groups studied the Young's modulus of the LC and parapapillary sclera as a measure of $\mathrm{ONH}$ stiffness. ${ }^{36-38}$ A common approach for the determination of Young's modulus is tensile testing of tissue strips. ${ }^{36,37}$ However, different techniques of measuring stiffness give different stiffness values, making a direct comparison of these values difficult. For instance, a stress-strain measurement showed Young's modulus of the LC was in the range of $E=11.8$ to 15.6 $\mathrm{MPa}$ and $E=28.5$ to $36.0 \mathrm{MPa}$ of the parapapillary sclera, ${ }^{36}$ whereas a Young's modulus of $17.2( \pm 2.7) \mathrm{kPa}$ was measured for the normal human LC using atomic force microscopy nanoindentation. ${ }^{38}$ To the best of our knowledge, this study 


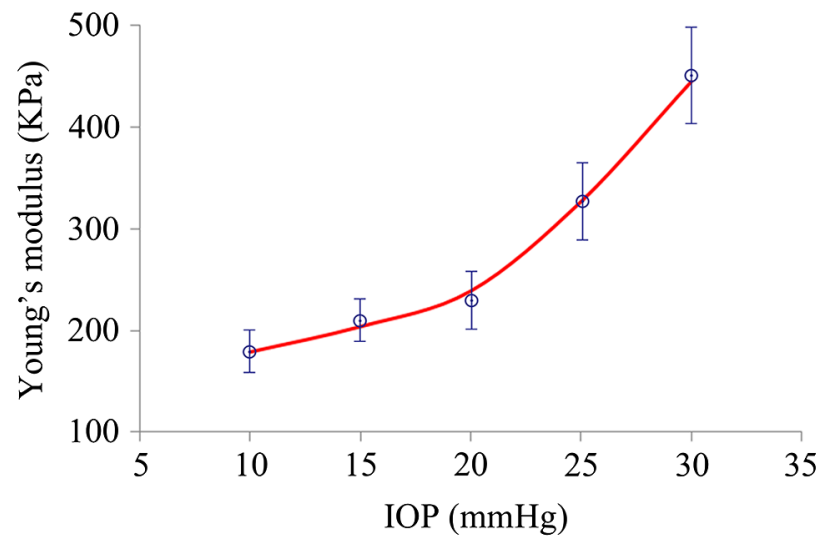

Fig. 6 The averaged Young's modulus of the ONH with elevated IOP. Error bars represent the deviation for each measurement. The curve fitting results show good agreement with a polynomial dependence to IOP.

is the first to apply OCE to analyze Young's modulus as a measure of stiffness in the ONH. As expected, Young's modulus measured reflects the more real values representative of whole eyes.

Although the quantification of the $\mathrm{ONH}$ biomechanical properties with elevated IOP has been demonstrated, there are still some challenges that need to be addressed for future applications. First, a faster imaging speed is required, especially for stiffer imaging objects. For this study, the imaging speed is sufficient to track the elastic wave propagation in the $\mathrm{ONH}$. But for the peripheral sclera, which is stiffer than the $\mathrm{ONH}$, our imaging system may not be fast enough to track wave propagation. A highspeed camera could help solve this issue. Second, the measured mechanical index of the current system is around 1.5, which exceeds U.S. Food and Drug Administration (FDA) regulations (0.23 in ophthalmology). Since, in this study, we acquired only a few hundred nanometers of axial displacement, ${ }^{32,39}$ the ultrasonic output can be reduced to meet the FDA regulations and maintain the ability to track elastic wave propagation. Third, ER is increased with elevated IOP. Since ultrasonic output remains unchanged in this study, the $\mathrm{ONH}$ became stiffer with elevated IOP, thus, the axial displacement decreased correspondingly. The signal-to-noise ratio may be decreased with a lower detected axial displacement, which leads to a high ER. ${ }^{40}$ However, the estimated elasticity is still in a reasonable range. Finally, we showed that it is feasible to reconstruct Young's modulus more accurately using $\mathrm{ONH}$ as the subject, however, the viscosity of the $\mathrm{ONH}$ with elevated IOP is not provided here. Tissue is predominantly elastic, with viscosity being typically much smaller as compared with elasticity. According to Eq. (2), when $\mu \gg \omega \eta$, the contribution of viscosity to overall viscoelasticity decreases as elasticity increases, curve fitting is unaffected by changes in viscosity. Moreover, the viscosity of the $\mathrm{ONH}$ is not important in this study, and to the best of our knowledge, there is currently no disease related to abnormal viscosity or alteration of viscosity.

\section{Conclusion}

In summary, we have demonstrated a method to quantify the biomechanics of the ONH with elevated IOP. The new method uses a confocal OCE system and realizes both high temporal and spatial resolution. We explored the feasibility of applying the Lamb wave model to reconstruct the biomechanics of thin- layered and viscoelastic $\mathrm{ONH}$. By fitting the phase dispersion curve with minimized $R^{2}$, the biomechanics of the ONH can be accurately determined. The study suggests that it should be feasible to evaluate biomechanics in vivo using the confocal configuration, which could lead to assessment of the biomechanics of the $\mathrm{ONH}$ in the clinic, allowing us to study the progression of glaucoma over time. Due to this advance, we believe that our confocal OCE system and methodology holds great potential for the detection of biomechanical properties in the posterior segment of the eye in clinical diagnosis.

\section{Disclosures}

No conflicts of interest, financial or otherwise, are declared by the authors.

\section{Acknowledgments}

Z.D. was supported by the Shandong Natural Science Foundation (Grant No. ZR2017MH055) as a visitor at Roski Eye Institute, University of Southern California. R.L. was supported by Provost Fellowship of University of Southern California. This work was partially supported by the National Eye Institute of the U.S. National Institutes of Health (NIH) under Grant Nos. R01EY028662, NIH P30EY029220, and an Unrestricted Departmental Grant from Research to Prevent Blindness, New York, NY 10017.

\section{References}

1. E. M. Van Buskirk and G. A. Cioffi, "Glaucomatous optic neuropathy," Am. J. Ophthalmol. 113(4), 447-452 (1992).

2. W. Wang et al., "Epidemiological variations and trends in health burden of glaucoma worldwide," Acta Ophthalmol. 97(3), e349-e355 (2019).

3. C. Burgoyne, "The morphological difference between glaucoma and other optic neuropathies," J. Neuro-Ophthalmol. 35(Suppl 1), S8-S21 (2015).

4. M. Hidalgo-Aguirre et al., "Automatic segmentation of the optic nerve head for deformation measurements in video rate optical coherence tomography," J. Biomed. Opt. 20(11), 116008 (2015).

5. A. J. Bellezza, R. T. Hart, and C. F. Burgoyne, "The optic nerve head as a biomechanical structure: initial finite element modeling," Invest. Ophthalmol. Vis. Sci. 41(10), 2991-3000 (2000).

6. J. Albon et al., "Age related compliance of the lamina cribrosa in human eyes," Br. J. Ophthalmol. 84(3), 318-323 (2000).

7. K. S. Yadav, R. Rajpurohit, and S. Sharma, "Glaucoma: current treatment and impact of advanced drug delivery systems," Life Sci. 221, 362-376 (2019).

8. Y. Hua, A. P. Voorhees, and I. A. Sigal, "Cerebrospinal fluid pressure: revisiting factors influencing optic nerve head biomechanics," Invest. Ophthalmol. Vis. Sci. 59(1), 154-165 (2018).

9. I. A. Sigal, J. G. Flanagan, and C. R. Ethier, "Factors influencing optic nerve head biomechanics," Invest. Ophthalmol. Vis. Sci. 46(11), 4189-4199 (2005).

10. E. Pavlatos et al., "Regional deformation of the optic nerve head and peripapillary sclera during IOP elevation," Invest. Ophthalmol. Vis. Sci. 59(8), 3779-3788 (2018).

11. B. Coudrillier et al., "Biomechanics of the human posterior sclera: ageand glaucoma-related changes measured using inflation testing," Invest. Ophthalmol. Vis. Sci. 53(4), 1714-1728 (2012).

12. Z. Wu et al., "Optic nerve head deformation in glaucoma: a prospective analysis of optic nerve head surface and lamina cribrosa surface displacement," Ophthalmology 122(7), 1317-1329 (2015).

13. C. F. Burgoyne et al., "The optic nerve head as a biomechanical structure: a new paradigm for understanding the role of IOP-related stress and strain in the pathophysiology of glaucomatous optic nerve head damage," Prog. Retinal Eye Res. 24(1), 39-73 (2005).

14. E. A. Sander et al., "A cellular solid model of the lamina cribrosa: mechanical dependence on morphology," J. Biomech. Eng. 128(6), 879-889 (2006). 
15. J. D. Pyne et al., "Sequential-digital image correlation for mapping human posterior sclera and optic nerve head deformation," J. Biomech. Eng. 136(2), 021002 (2014).

16. J. Ophir et al., "Elastography: a quantitative method for imaging the elasticity of biological tissues," Ultrason. Imaging 13(2), 111-134 (1991).

17. X. Qian et al., "Multi-functional ultrasonic micro-elastography imaging system," Sci. Rep. 7(1), 1230 (2017).

18. X. Qian et al., "Ultrasonic microelastography to assess biomechanical properties of the cornea," IEEE Trans. Bio-Med. Eng. 66(3), 647-655 (2019).

19. M. A. Kirby et al., "Optical coherence elastography in ophthalmology," J. Biomed. Opt. 22(12), 1-28 (2017).

20. Z. Han et al., "Optical coherence elastography assessment of corneal viscoelasticity with a modified Rayleigh-Lamb wave model," $J$. Mech. Behav. Biomed. Mater. 66, 87-94 (2017).

21. C. Wu et al., "Assessing age-related changes in the biomechanical properties of rabbit lens using a coaligned ultrasound and optical coherence elastography system," Invest. Ophthalmol. Vis. Sci. 56(2), 1292-1300 (2015).

22. V. Y. Zaitsev et al., "Optical coherence elastography for strain dynamics measurements in laser correction of cornea shape," J. Biophotonics 10(11), 1450-1463 (2017).

23. J. Li et al., "Differentiating untreated and cross-linked porcine corneas of the same measured stiffness with optical coherence elastography," J. Biomed. Opt. 19(11), 110502 (2014).

24. Z. Han et al., "Quantitative methods for reconstructing tissue biomechanical properties in optical coherence elastography: a comparison study," Phys. Med. Biol. 60(9), 3531-3547 (2015).

25. L. Ambrozinski et al., "Acoustic micro-tapping for non-contact 4D imaging of tissue elasticity," Sci. Rep. 6, 38967 (2016).

26. D. Jia et al., "Experimental evaluation of surface topographies of NMQL grinding $\mathrm{ZrO}_{2}$ ceramics combining multiangle ultrasonic vibration," Int. J. Adv. Manuf. Technol. 100(1-4), 457-473 (2019).

27. S. Chen, M. Fatemi, and J. F. Greenleaf, "Quantifying elasticity and viscosity from measurement of shear wave speed dispersion," J. Acoust. Soc. Am. 115(6), 2781-2785 (2004).

28. Y. Qu et al., "In vivo elasticity mapping of posterior ocular layers using acoustic radiation force optical coherence elastography," Invest. Ophthalmol. Vis. Sci. 59(1), 455-461 (2018).

29. I. Z. Nenadic et al., "Phase velocities and attenuations of shear, Lamb, and Rayleigh waves in plate-like tissues submerged in a fluid (L)," J. Acoust. Soc. Am. 130(6), 3549-3552 (2011).

30. T. M. Nguyen et al., "Assessment of viscous and elastic properties of sub-wavelength layered soft tissues using shear wave spectroscopy: theoretical framework and in vitro experimental validation," IEEE Trans. Ultrason. Ferroelectr. Freq. Control 58(11), 2305-2315 (2011).

31. C. C. Shih et al., "Quantitative assessment of thin-layer tissue viscoelastic properties using ultrasonic micro-elastography with Lamb wave model," IEEE Trans. Med. Imaging 37(8), 1887-1898 (2018).

32. J. Zhu et al., "Imaging and characterizing shear wave and shear modulus under orthogonal acoustic radiation force excitation using OCT Doppler variance method," Opt. Lett. 40(9), 2099-2102 (2015).

33. Y. Qu et al., "Acoustic radiation force optical coherence elastography of corneal tissue," IEEE J. Sel. Top. Quantum Electron. 22(3), 6803507 (2016).

34. Y. He et al., "Confocal shear wave acoustic radiation force optical coherence elastography for imaging and quantification of the in vivo posterior eye," IEEE J. Sel. Top. Quantum Electron. 25(1), 1-7 (2018).

35. Y. Qu et al., "Quantified elasticity mapping of retinal layers using synchronized acoustic radiation force optical coherence elastography," Biomed. Opt. Express 9(9), 4054-4063 (2018).

36. E. Spoerl, A. G. Boehm, and L. E. Pillunat, "The influence of various substances on the biomechanical behavior of lamina cribrosa and peripapillary sclera," Invest. Ophthalmol. Vis. Sci. 46(4), 1286-1290 (2005).

37. A. Eilaghi et al., "Biaxial mechanical testing of human sclera," J. Biomech. 43(9), 1696-1701 (2010).

38. C. Braunsmann et al., "Evaluation of lamina cribrosa and peripapillary sclera stiffness in pseudoexfoliation and normal eyes by atomic force microscopy," Invest. Ophthalmol. Vis. Sci. 53(6), 2960-2967 (2012).
39. J. Zhang et al., "High-dynamic-range quantitative phase imaging with spectral domain phase microscopy," Opt. Lett. 34(21), 3442-3444 (2009).

40. E. Maksuti et al., "Arterial stiffness estimation by shear wave elastography: validation in phantoms with mechanical testing," Ultrasound Med. Biol. 42(1), 308-321 (2016).

Zhaodong Du received his $\mathrm{PhD}$ in ophthalmology from Peking University in Beijing, China, in 2010, under Dr. Zhizhong Ma. Following surgery training at the Affiliated Hospital of Qingdao University, Qingdao, China, he became a retinal surgeon in the Affiliated Hospital of Qingdao University and an associate professor at Qingdao University, in 2016. He is currently a visiting scholar at Roski Eye Institute, University of Southern California, California, United States.

Runze $\mathbf{L i}$ received his BS degree in biomedical engineering from Huazhong University of Science and Technology, Wuhan, China, in 2014, and MS degree in biomedical engineering from the University of Southern California, Los Angeles, California, in 2017. $\mathrm{He}$ is currently a PhD student at the Department of Biomedical Engineering, University of Southern California. His research interests include the development of high frequency transducer, ultrasonic elastography, and optical coherence elastography.

Xuejun Qian received his BS degree in electrical engineering from Xidian University, China, in 2012. He received his MS degree in electrical engineering and $\mathrm{PhD}$ in biomedical engineering from the University of Southern California, Los Angeles, California, United States, in 2014 and 2018, respectively. He joined the NIH Ultrasonic Transducer Resource Center (UTRC) as a research assistant under the supervision of Dr. Q.Z. and Dr. K. Kirk Shung. His research interests include high-frequency ultrasound elastography, optical coherence elastography, super-resolution microvessel imaging, and multimodality imaging.

Gengxi Lu received his bachelor's degree in physics from Nanjing University, China, in 2017. He is currently a PhD student at the Department of Biomedical Engineering, University of Southern California, Los Angeles, California, United States. His research interests include the development of intravascular ultrasound transducers, photoacoustics, ophthalmology, and acoustic metamaterials.

Yan $\mathbf{L i}$ received her BS and MS degrees from Tianjin University and Changchun Institute of Optics, Fine Mechanics and Physics, Chinese Academy of Sciences, Beijing, China, in 2011 and 2014, respectively. She is currently working toward her $\mathrm{PhD}$ under the guidance of $\mathrm{Dr}$. Z.C. Her research interests include multimodality endoscopic imaging by combining ultrasonic and optical techniques, such as ultrasound, optical coherence tomography, photoacoustic imaging, and fluorescence.

Youmin He received his BS and MS degrees from the School of Electronics and Information Engineering, Beijing Jiaotong University, Beijing, China, in 2011 and 2014, respectively, and his MS degree in biomedical engineering from the University of California, Irvine, California, United States, in 2017. He is currently working toward his $\mathrm{PhD}$ degree under the guidance of Dr. Z.C. His research interests include fast image segmentation, parallel computing for real-time OCT imaging, high-resolution OCT system for airway imaging, and ARF-OCE technology development and application in ophthalmology and cardiology.

Yueqiao Qu received her BS degree in biomedical engineering from Johns Hopkins University, Baltimore, Maryland, United States, and her MS degree in biomedical engineering from the University of California, Irvine, California, United States, in 2011 and 2015 , respectively. She is currently working toward her PhD under the guidance of Dr. Z.C. Her research interests include ARF-OCE technology development and applications in ophthalmology and cardiology, integrated intravascular ultrasound (IVUS) and OCT imaging, and miniature optical fiber catheter development for photoacoustic imaging, IVUS-OCT, and ARF-OCE. She was the recipient of the National Institutes of Health T32 fellowship and the F31 grant. 
Laiming Jiang received his BS degree in materials science and engineering from Sichuan University, China, in 2013. He is currently a joint PhD student from Keck School of Medicine at the University of Southern California, Los Angeles, California, and the College of Materials Science and Engineering, Sichuan University, Sichuan, China. His current research interests include the development of lead-free piezoelectric materials and flexible ultrasonic devices.

Zeyu Chen received his BE degree from Central South University, China, in 2012. He is currently a postdoctoral research associate in the Department of Biomedical Engineering at the University of Southern California (USC) under the direction of Dr. Q.Z. and Dr. $\mathrm{K}$. Kirk Shung. $\mathrm{He}$ is conducting his research in the NIH Ultrasonic Transducer Resource Center (UTRC) on ultrasonic transducer fabrication and additive manufacturing of functional materials.

Mark S. Humayun received his bachelor of science degree from Georgetown University in 1984, his master's degree from Duke University in 1989, and his PhD from the University of North Carolina, Chapel Hill, in 1994. He completed his ophthalmology residency at Duke Eye Center and fellowships in both vitreoretinal and retinovascular surgery at Johns Hopkins Hospital. He stayed on as a faculty at Johns Hopkins, where he rose to the rank of associate professor before moving to USC in 2001. He has devoted much of his career to clinical and scientific research in ophthalmology and bioengineering, becoming both a biomedical engineer and professor of ophthalmology. He led a talented and diverse team of interdisciplinary researchers with the ultimate goal of creating the world's first artificial retina. $\mathrm{He}$ assembled a team of world experts with a wide range of proficiency, including biomedical engineering, computer science, medicine, chemistry, biology and business. In clinical trials since 2007 and approved by the FDA in 2013, the Argus II retinal implant represents the culmination of a visual restoration strategy that offers an unprecedented degree of sight to those with complete retinal blindness. He was elected to the prestigious National Academy of Medicine (NAM) and National Academy of Engineering (NAE) for his pioneering work to restore sight. With over 200 publications and more than 100 patents and patent applications, he has received several research awards, including the 2005 Innovator of the Year award. He was also featured as one of the top 10 inventors in Time magazine in 2013, voted as one of the best doctors in America for three years, and one of the top $1 \%$ of doctors by U.S. News \& World Report. In 2016, he received the National Medal of Technology and Innovation from President Barack Obama for his innovative work and development of the Argus II.
Zhongping Chen received his BS degree in applied physics from Shanghai Jiao Tong University, Shanghai, China, in 1982. He received his MS degree in electrical engineering and $\mathrm{PhD}$ in applied physics, both from Cornell University, Ithaca, New York, United States, in 1987 and 1993, respectively. He is currently working as a professor of biomedical engineering and the director of the F-OCT Laboratory at the University of California, Irvine, California, United States. He is a cofounder and board chairman of OCT Medical Imaging, Inc., Irvine, California. His research interests include the areas of biomedical photonics, microfabrication, biomaterials, and biosensors. His research group has pioneered the development of functional optical coherence tomography, which simultaneously provides high-resolution three-dimensional images of tissue structure, blood flow, and birefringence. He has published more than 220 peer-reviewed papers and review articles and holds a number of patents in the fields of biomaterials, biosensors, and biomedical imaging. $\mathrm{He}$ is a fellow of the American Institute of Medical and Biological Engineering, a fellow of SPIE, and a fellow of the Optical Society of America.

Qifa Zhou received his PhD from the Department of Electronic Materials and Engineering, Xi'an Jiaotong University, Xi'an, China, in 1993 . He is currently working as a research professor with the NIH Resource on Medical Ultrasonic Transducer Technology and the Department of Biomedical Engineering and Industry and System Engineering, University of Southern California (USC), Los Angeles, California, United States. Before joining USC in 2002, he worked in the Department of Physics at Zhongshan University in China, the Department of Applied Physics at Hong Kong Polytechnic University, and the Materials Research Laboratory at Pennsylvania State University. His current research interests include the development of ferroelectric thin films, MEMS technology, nanocomposites, and modeling and fabrication of high-frequency ultrasound transducers and arrays for medical imaging applications, such as photoacoustic imaging and intravascular imaging. He has published more than 130 journal papers in this area. He is a fellow of the International Society for Optics and Photonics and the American Institute for Medical and Biological Engineering. $\mathrm{He}$ is also a senior member of the IEEE Ultrasonics, Ferroelectrics, and Frequency Control (UFFC) Society and a member of the UFFC Society's Ferroelectric Committee. He is a member of the Technical Program Committee of the IEEE International Ultrasonics Symposium. He is an associate editor of IEEE Transactions on Ultrasonics, Ferroelectrics, and Frequency Control. 